\title{
Reply to comment on "Ambiguity about the volume of colloids administration in a clinical study of thoracic esophagectomy precludes conclusion on renal safety of hydroxyethyl starch"
}

\author{
Takeo Fujita ${ }^{1}$. Naoya Okada ${ }^{1}$ ' Yasumasa Horikiri ${ }^{1}$ Takuji Sato $^{1} \cdot$ Hisashi Fujiwara ${ }^{1}$. Shuhei Mayanagi ${ }^{1}$. \\ Jun Kanamori ${ }^{1} \cdot$ Hiroyuki Yamamoto $^{1} \cdot$ Hiroyuki Daiko $^{1}$
}

Received: 31 March 2019 / Accepted: 1 July 2019 / Published online: 15 July 2019

(c) Springer Nature Singapore Pte Ltd. 2019

We thank Dr. C. J. Wiedermann for his interest in our paper [1], which investigated the safety and efficacy of hydroxyethyl starch 6\% 130/0.4/9 solution versus 5\% human serum albumin in thoracic esophagectomy with three-field lymphnode dissection. The results of our study suggested that hydroxyethyl starch 6\% 130/0.4/9 was well tolerated and comparable to human serum albumin in its effect on renal function during thoracic esophagectomy, with no obvious adverse event, particularly, elevation of serum creatinine levels.

We agree with Dr. Wiedermann's valuable point about our mistake in this manuscript. In our study, $1000 \mathrm{ml}$ of hydroxyethyl starch 6\% 130/0.4/9 solution was administered during the thoracic phase of the procedure, at $150-250 \mathrm{ml} / \mathrm{h}$, dependent on the patient's status and operative duration, in comparison with $1000 \mathrm{ml}$ of human serum albumin administered in the same way.

Dr. Wiedermann's observation was invaluable for correcting this detail in our paper.

\section{Compliance with ethical standards}

Conflict of interest We have no conflicts of interest or financial ties to disclose.

\section{Reference}

1. Wiedermann CJ. Ambiguity about the volume of colloids administration in a clinical study of thoracic esophagectomy precludes conclusion on renal safety of hydroxyethyl starch. Surg Today. 2019. https://doi.org/10.1007/s00595-019-01798-6.

Publisher's Note Springer Nature remains neutral with regard to jurisdictional claims in published maps and institutional affiliations.
Takeo Fujita

takfujit@east.ncc.go.jp

1 Division of Esophageal Surgery, National Cancer Center Hospital East, 6-5-1 Kashiwanoha, Kashiwa 277-8577,

Chiba, Japan 